\title{
Design and Implementation of Visual CSCL Collaborative Knowledge Building Tool
}

\author{
Jian-feng Ren, Shuang Liang, Hua Guo \\ Department of Education Technology, Capital Normal University, Beijing, China \\ rjfkcyx@163.com,ghsj456@163.com
}

\begin{abstract}
The popular CSCL system based on B/S architecture has got a good development, but these kind of systems have fundamental defects in Visualization, graphical, and interactive experience speed etc. silverlight is a appropriate choice to solve the above problems, and the system based on silverlight works more effective in supporting Collaborative learning with the strategies for collaborative knowledge building. This paper explored the technique and method to apply Silverlight to CSCL tools based on a sets of strategies, then discussed the support theory, development ideas. Finally, the process to design and develop a sample tool is also discussed in the paper. The tool has more advantages in supporting collaborative knowledge building.

Index Terms - CSCL, knowledge building, visualization .
\end{abstract}

\section{Instruction}

This paper aims to use Silverlight which is highly interactive and rich user experience RIA (Rich Internet Applications)technology to design and develop a visual CSCL learning collaborative tool. Though this tool to improve learners' knowledge construction level in learner group online discussion and collaborative creation .

\section{A. Research Background and Questions}

Try As a new way to maximize the individual and group performance and to improve the quality of formal and informal learning, CSCL pursuits collaborative knowledge building mediated by technology. The collaborative knowledge building through the learner interaction and negotiation, mutual inspiration, encouragement and support, the meaning of generation of knowledge especially in common understanding of learning content, experience harvest and success and get the common development.

At present, there are many special CSCL tools support collaborative knowledge construction to some extent. However most of the the realization of CSCL tools are based on B/S model, the HTML page and the server data transfer mode. There are several issues as follows.

1) Paper the Limitations of the Technology Itself: The pressure on the server end data processing, lack of the ability to deal with the rich user interface, and refresh the page frequently, the learners' experience is bad. And because of the lack of social cues in remote CSCL, learners in collaborative learning , basically depend on the text clues, then there are a lot of limitations in mutual understanding and coordination.

2) The Problem of Interactive Support Strategy: The existing platforms pay more attention to the realization of the main function of information technology, but their support and focus on collaborative strategy is not enough. I once pointed out, simply put learners in a computer communication environment with common learning task, does not guarantee an effective collaborative learning[1]. It is difficult for learning community members to maintain effective interaction spontaneously. There is often some learners have been speaking, some learners never speak. Speakers tend to make conclusive statements, listener without debate, discuss which lead to low level of collaboration, poor effect and some other bad results. The lack of effective collaboration strategy support, reaction in the corresponding platform, will make the corresponding platform in addition to learning group, achievement statistics thereof, and there is no much difference in general communication space.

3) Problems of Design: Most collaborative learning tools don't show who is the speaker intuitively, and require learners to identify through words. these tools don't show the speaker's attitude intuitively, and can't lead the occurrence of interaction and move forward. In addition, the interface is not in conformity with the learner's cognitive characteristics, text layout is overmuch, lack of visualization, graphical design and so on, are also greatly affects the learners' interaction and personal meaning construction.

\section{The Supporting Theories and Requirements of Collaborative Knowledge Construction}

Collaborative knowledge construction as the core pursuit of CSCL, there are various theoretical basis. Here only take the constructivism learning theory and the situated cognition theory for example to discuss briefly .

Piaget and some others think that social cognitive conflict when people are co-produced in an environment can cause cognitive imbalances that in turn stimulate people's perspective-taking ability and cognitive development[2].In CSCL, the ongoing communication between students Constantly create cognitive imbalance and power of balanced development will actively promote promote students' positive view comparison, demonstration, explanation, correction and other activities to support the continuous development of cognitive.

Vygotsky believed cognitive development produce in social interaction. Between cognitive development, each mental function have occurred twice, the first time in people to participate in social interaction, the second in the individual psychological level, the individual inside. Children initially completed cognitive tasks in support of the social partners, later times, these social interaction will gradually be internalized until they are able to complete the task by themselves. Therefore, the individual psychological processes 
originated in social interaction. To illustrate this process, Vygotsky proposed the concept of zone of proximal development. ZPD refers to the gap between children able to complete tasks independently and in interaction with adults or better peers to complete tasks. Vygotsky believed that the most important feature of the teaching is it creating the zone of proximal development. This creates a greater likelihood of children continuous development. Mental function regulating by language and other cultural tools. Culture shapes and determines human behavior, cultural norms and others affect learning opportunities for children[3].

According to vygotsky's account, the synergistic effect CSCL are counting on, is the effect of the zone of proximal development. And the corresponding tools adjust the learning process. Collaboration, basically can be seen as a process of Co Construction of meaning, the meaning construction can not be seen as the mental representations of individual participants, but the result of the people interaction. Take, for example, people turn to speak. At this time, the speech is different from the teacher let students speak alone, and meaning is not generated by a single student to speak, because it usually relies on references to shared context, to speak after the recessive inheritance, but also set the tone for the subsequent statement[4].

The theory of Situated Cognition not only think learning as a construction process, at the same time also believe that what those students construct in the community construction not only the "personalized" understand to the outside world, but also construct "identity", construct their role and influence in society, to obtain recognition and the realization of individual value. Learning is, as it were, "meaning" and "identity" of twofold construction process.

The theories above put forward higher requirements for CSCL support system. Corresponding system shall be able to smooth, quick and well support learners share, search, picking, display, inquiry, interpretation, produce cognitive conflict, negotiation, forming a Shared understanding or achievements, the development of sharing achievement and other knowledge construction activities. In addition, in the environment of remote not face to face, still need through the visualization, the design of visualization and the corresponding strategy, rebuild "present" feeling, make the "identity" construction also obtain good support.

In contrast, the current popular, especially the domestic collaborative learning support system, the text of interface and the lack of effective strategies, makes it more difficult to achieve the above requirements. The solution of the problem, on the one hand, depends on the research progress of where is "associate" of CSCL collaborative knowledge construction support strategy, on the other hand, The emergence of new information technology provide the possibility for a relevant strategy better embedded in the system.

\section{A Design Idea for Visualization Collaborative Knowledge Construction Tool}

In recent years, we have done a systematic study in CSCL collaboration support strategy system, the organization strategy of specific activities and its underlying principles, such as micro-policy interaction speech and research to develop appropriate policy embedded in the ordinary and the use of RIA technologies such as specialized CSCL tool. And research how to embed corresponding strategies into the ordinary and specialized tools such as CSCL developed by RIA technologies[1][5][6][7][8].RIA and other technologies enable the corresponding tools have a rich interface experience effects, and achieve the client asynchronous data transmission and processing. While reduce the server load, also reduce the response time of the user interface, eliminate the blank waiting caused by frequently click on traditional Web pages, improve the efficiency of interaction, but also make it easier to achieve graphical real-time interactive function, which will bring great improvements to collaborative knowledge construction in remote CSCL system based on B/ $\mathrm{S}$ model .

In the tool design, in order to promote learners 'meaning' and 'identity' dual construction, we let learners can see the image of who participated in the collaborative and coordinated progress. This first need to allow learners "sense" the presence of other learners to form a stronger sense of presence, mobilize active participation.

Research indicates that when learners perceive having a companion around the speech will be more active, it is necessary for efficient collaboration. Y. CAO and J. GREER think that the Internet provides a convenient and comfortable learning environment, but it did not deal with the learners will feel "lonely, deaf and dumb" sort of problem[9]. "Perception" is the learners get if other collaborators are in beside, whether it will work, what they are doing, where they are and so on .In CSCL, the "perception" is necessary for the efficient collaboration, plays an important role, especially in collaboration by the debate, arguing. Y. CAO and others' experimental data indicate that the "perception" enable students to acquire the needed resources (human or electronic resources) more convenient and easy; in the support of "perception", students can better participate in their work[9]."Perception" tool supports collaborative learning by facilitating information exchange and strengthen communication in virtual space among students. Therefore, when there are learners come to participate in group learning, it is necessary to show his presence at the first time.

Secondly, we need to strengthen the learner's attitude performance during the exchange, promote the improvement of the degree of learner participation in the exchange.

In the collaborative learning process, if a statement of learners got a clear attitude feedback will greatly facilitate the level of interaction in the learning process and continues of learning activities. On the one hand, the other speakers need to compare the speaker's information and their own cognitive structures to get the cognitive conflict feedback to give a distinctive attitude feedback to the speaker. On the other hand, after obtaining feedback the speaker needs to refute or to continue to explain, feedback need interacts with cognitive structure ; this will also facilitate the exchange of feedback and extends the entire communication activities and improve the 
interactive level and individual construct level.

This objective will also contribute to role orientation of learners themselves as opponents, supporters, neutral , social psychologists believe that if lack of role awareness, their role is not clear, do not understand other people's thoughts and feelings, will fail in the activity. All these enlighten us to orientate role, clear statements attitude.

Third, the design of reminders to guide the participation learners who is not active if necessary. Because learners dispersed throughout the network, they are often in a "latent" state, only obtaining the information provided by other learners, and their genuine participation rarely, which not only make their own meaning construction at a low level, but also reduce the level of the entire group interactive learning activities. So, it is necessary to design personalized guidance function depending on the learner's performance.

\section{The Design and Implementation of the Visualization CSCL Collaborative Knowledge Construction Gadget}

\section{A. The client implementation}

The Silverlight controls in the tool is responsible for the display of human-computer interaction with learners directly in the exchanges and cooperation process, so it is very important .The overall Silverlight controls interface we design shown in Fig 1:

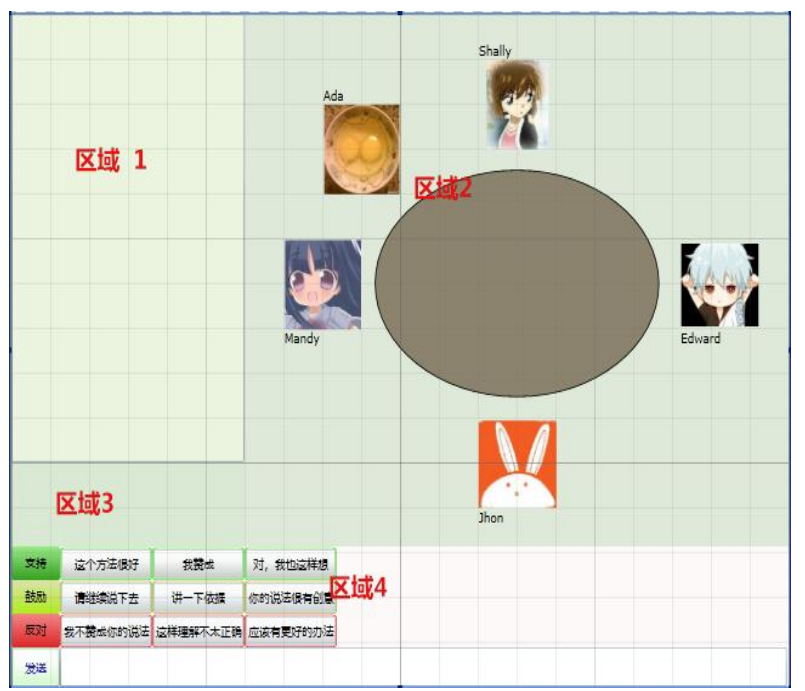

Fig. 1 Collaborative interface design

The interface is divided into four regions. Zone 1 is in text form used to shows the interaction of learners, including the speakers' names, content, and their speak attitude tendency and time. Area 2 is the main display area. Visualize display the interactions between online panel members. When panel members speaking, there will be text display at the current location of team members, which according to different attitudes displayed with different colors. Zone 3 is the notification area. If participants did not speak for a long time (eg 3 minutes) then given text reminder: "You have not spoken for a long time, please pay attention to other people's speech and express your views."; In addition, when participants only in a single perspective, such as continuous favor but without their own opinions or views, we design sentences similar to "You have commend someone ten times in a row, please publish some of your opinions," and "Could you think about it from another angle ? "and so prompt to guide. Region 4 to the input region, comprises two parts. One part is short statements based on pre-set network micro interactive language which has a clear stand, and you can send it out just by a click[7].In order to simplify the development difficulty, here the micro-interactive speech and languagerelated guidance proposed by the author were simplified. The speaker's attitude is just simply divided into three kinds: against displayed in red; favor, then green; encourage or neutral, is displayed in yellow. Another part is the user-input. The default attitude is neutral, so user input is displayed in yellow. If users need to change their own speak attitude, just beginning with the "/ $\mathrm{r}$ " to indicate the opposition or beginning with "/g" expressed support .

Page layout controls in a nested manner. Outermost layer using Grid to frame a given range, the Grid is internally divided four zones. Because of areas 1 and 3 are relatively independent, each with a separate control to complete; Zone 2 with Canvas layout; area 4 Uses Stackpanel to layout. So we can get every student's information in corresponding zone.

\section{B. The server-side and implementation of the database}

The role of the server in the whole system is to maintain a list of online learners, record information of the user's speech; according to these requests information to reply, and to communicate with databases. Server-side set up a chat engine that is run when the server starts, records the transfered information from various users, providing modify online users list, stores and queries the user information on the ways and means to speak.

In CSCL, the interaction analysis is the core function to support collaborative and evaluation. Interactive analysis relies on the behavior information gathering of the participants in the collaborative process. This information includes a variety of data types and formats. Therefore, we need for a flexible, standardized data format. In this system, the server uses SQL server express 2008. We designed three tables to store data. The first is user information table for storing registered users' basic information, such as user name, password, avatar picture storage location, nickname, etc; the second is the active sheet, recording the basic information of activities, such as the number of events, dates, participants, etc. information; third is session table, recording each activity when someone made a statement, spoken content. This format can record information as the data needed and widely adapt to a variety of information-gathering activities.

\section{The using Effect}

Fig. 2 is the region 2 when this tool used in the process of collaborative learning, i.e., a schematic diagram of the main display area. Participants in personal image sitting around a round table, each spoke appears at the side of their image and attitude their holdings of expressed by different colors. 
Guidance information and all interactions progress are also displayed in reminded area and text area.

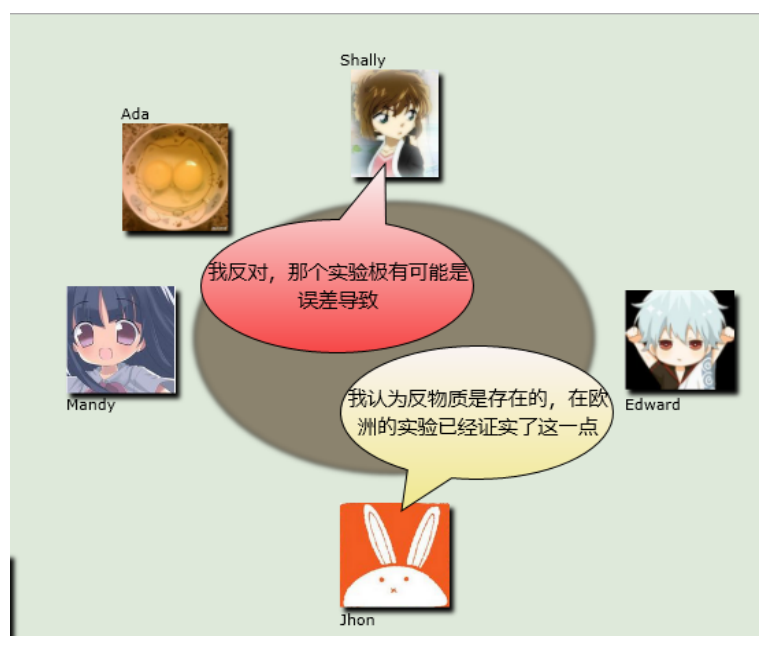

Fig. 2 Virtual Roundtable discussion

This paper describes a remote CSCL Visualization Collaborative Knowledge construction professional tool based on appropriate policy. The tool's purpose is to support more effective interaction and depth of collaboration, display the collaborative process and the attitude of the learner with visual forms. It clearly perceive the presence of fellow learners; clearly show the attitude of peers and their own attitude to companions' statements ;promote the development of learning exchange process; enhance the level of group interaction and increase the effect of learners promote the depth of collaborative knowledge construction.

The system uses silverlight technology to improve human-computer interaction, make the interface intuitive and friendly, more interactive features; enhance the participation of the students 'presence' feeling, so that it can visually see each learner in knowledge sharing, display, question, interpret, negotiation and other collaborative knowledge construction activities; show the processes and learners' performance in every important part; and more intuitively understand their "identity" construction process, is a good supplement to a simple interface CSCL system. In addition, which also embedded simplifies micro-interactive speech and prompt, they played a guiding role for discussion learners .

The tool also has many deficiencies, in learners' behavior records and more effective guiding, and the interface more real and vivid, etc. are inadequate, we will continue to improve.

\section{Conclusion}

This paper describes a remote CSCL Visualization Collaborative Knowledge construction professional tool based on appropriate policy. The tool's purpose is to support more effective interaction and depth of collaboration, display the collaborative process and the attitude of the learner with visual forms. It clearly perceives the presence of fellow learners; clearly shows the attitude of peers and their own attitude to companions' statements; promotes the development of learning exchange process; enhances the level of group interaction and increase the effect of learners promote the depth of collaborative knowledge construction.

The system uses silverlight technology to improve human-computer interaction, make the interface intuitive and friendly, more interactive features; enhance the participation of the students 'presence' feeling, so that it can visually see each learner in knowledge sharing, display, question, interpret, negotiation and other collaborative knowledge construction activities; show the processes and learners' performance in every important part; and more intuitively understand their "identity" construction process, is a good supplement to a simple interface CSCL system. In addition, which also embedded simplifies micro-interactive speech and prompt, they played a guiding role for discussion learners .

The tool also has many deficiencies, in learners' behavior records and more effective guiding, and the interface more real and vivid, etc. are inadequate, we will continue to improve.

\section{Acknowledgment}

The paper is supported by national education science plan project "research on the support strategies and the system of visual CSCL collaborative knowledge building"(project No.DCA120189).

\section{References}

[1] REN Jian-feng, Strategy research to promote the remote CSCL interactions - The new issue of CSCL research, Audio Visual Education Research, tenth period in 2007.

[2] David W. Johnson and Roger T. Johnson, cooperation AND the technical application, J. Michael Spector $\backslash$ M. David Merrill 《Handbook of research on educational communication and technology $\rangle$ page 450,East China Normal University press, 2012.

[3] Robert Ziegler, 《The development of children's thinking》 page 122, The World Book Publishing House,2006.

[4] R. Keith Sawyer. 《The Cambridge Handbook of the Learning Sciences 》page 416,Education Science Press,2010.

[5] REN Jian-feng, The research of organizational strategy of remote CSCL interactive activities and corresponding system with step by step discussion model, Chinaaudio-visual education, Eighth period in 2008.

[6] REN Jian-feng, FANG Hai-guang, LI Jian-zhu, LUO Jian, The research of organizational strategy of remote CSCL interactive activities and corresponding system with the role discuss model, The 2007 Education Technology Doctoral Forum.

[7] REN Jian-feng, Micro interactive speech in network collaborative learning, The modern education technology, Ninth period in 2010 .

[8] GUO Li-na, REN Jian-feng, Design and implementation of visual collaborative knowledge construction tools based on Silverlight' Audio Visual Education Research, fourth period in 2012.

[9] Y. CAO \& J. GREER .Supporting awareness to facilitate collaborative learning in an online learning environment.Educational Technology \& Research 2002(1):9-18 